\title{
Comparison on Identification of Affective Body Motions by Robots Between Elder People and University Students: A Case Study in Japan
}

\author{
Tatsuya Nomura • Akira Nakao
}

Accepted: 5 March 2010 / Published online: 18 March 2010

(c) The Author(s) 2010. This article is published with open access at Springerlink.com

\begin{abstract}
Expressive behaviors based on body motions are one of the useful methods that social robots present their emotional states toward users. On the other hand, some psychological research found age dependence on emotion identification in human facial expressions. In order to investigate this dependence in affective body expressions of robots, a psychological experiment was conducted in Japan, by using a small-sized humanoid robot on which three types of affective motion expression (anger, sadness, and pleasure) were implemented. The results of the experiment, which consisted of seventeen university student subjects and fifteen elder subjects, showed differences between younger and elder subjects on emotion identification, body parts paid attention to, and impressions of motion speed and magnitude for these affective body motions of the robot. Moreover, the results suggested correlations between the accuracy of emotion identification and cognitive bias to the robot's specific body motion parts. Based on these results, the paper discusses about some implications in human-robot interaction research.
\end{abstract}

T. Nomura $(\bowtie)$

Department of Media Informatics, Ryukoku University,

1-5, Yokotani, Seta-ohe-cho, Otsu, Shiga 520-2194, Japan

e-mail: nomura@rins.ryukoku.ac.jp

url: http://www.rikou.ryukoku.ac.jp/ nomura/

T. Nomura

ATR Intelligent Robotics and Communication Laboratories, 2-2-2 Hikaridai, Keihanna Science City, Kyoto 619-0288, Japan e-mail: nomura@atr.jp

A. Nakao

Graduate School of Science and Technology, Ryukoku University,

1-5, Yokotani, Seta-ohe-cho, Otsu, Shiga 520-2194, Japan

e-mail: t09m076@mail.ryukoku.ac.jp
Keywords Emotion - Body motion expression · Elderly · Psychological experiment

\section{Introduction}

Expressive behaviors based on body motions are one of channels for communication between humans. It has a possibility of contribution to human-robot interaction, in particular, affective information from sociable robots to humans [2]. In fact, several researchers proposed and validated mechanisms of body motion expressions in robots.

Nakata, Mori, and Sato [11] proposed a set of physical values for robot motions based on a theory of body movement psychology, and experimentally found correlations between these physical values and human impressions of the corresponding affective body motions expressed by a small-sized robot. Itoh et al. [5] developed a humanoid robot that could express several emotions including anger, sadness, and happiness, based on body motions. Marui and Matsumaru [9] explored motion expressions of three basic emotions, pleasure, sadness, and anger, by using a teddy bear robot. Recently, Yuk and Kwon [16] developed a legwheel mobile robot which could conduct various types of body motions, and validated the effectiveness of the proposed emotional body motions consisting of happiness, sadness, and anger. Moreover, Kim, Kwak, Han, and Kwak [8] developed a robot with a simple body, two eyes, and fullcolor light emitting diodes, and evaluated its emotion expressions based on colors by combining with verbal expressions.

However, these existing studies adopted university students or younger adults as evaluators, and lacked the consideration of user demographics in the experiments. Some psychologists and sociologists argue that interpretation of 
emotional expressions is dependent on societies and cultures $[3,6]$. Thus, it is estimated that the effectiveness of affective body motions expressed by robots depend on users' demographic factors such as gender and age. Age dependence should particularly be investigated in the current situation that human-robot interaction is expected as one of assistive technologies in home for the elderly in industrialized countries including Japan, due to the decrease in rates of childbirth and the increase in the elderly population.

On age dependence of emotion identification, there is an important study in neuropsychology. Wong, CroninGolomb, and Neargarder [17] suggested age differences on emotion identification for facial expressions. Their experimental study based on photo stimuli revealed that older participants were worse at identifying the emotions of anger, fear, and sadness than were younger participants, although the ability to identify accurately the emotions of happiness and surprise were comparable between the two participant groups. Moreover, it found several differences on eye movement patterns between these participant groups: older participants made fewer fixations on faces compared with younger participants, and made a greater proportion of fixations to the lower halves of faces compared with younger participants. Furthermore, correlation analysis between these fixations and the accuracy of emotion identification suggested that the tendency to fixate more on the lower half of the face adversely affected the older participants' ability to identify certain emotions. Referring to the fact that accurate identification of fearful, angry, and sad faces requires examination of the upper half of the face, Wong et al. [17] suggested that older participants were at a distinct disadvantage on identifying these three facial expressions by fixating on the lower halves of faces.

The above study by Wong et al. [17] has an important implication. Even if designers implement affective body expressions of robots and they are interpretable for younger users, there is a possibility that older users fail to identify the intended emotions. Moreover, the accuracy of emotion identification may be affected by cognitive characteristics such as visual bias to specific parts of robot motions not required for the identification.

Thus, the paper focuses on the following research questions

1. Are there differences on emotion identification of body motions expressed by robots between younger and elder people?

2. Are there correlations between the accuracy of this emotion identification and cognitive bias to robots' specific body motion parts?

In order to investigate these questions, a psychological experiment was conducted in Japan. This was a case study in the sense that all the participants were Japanese. In this experiment, some affective body motions were implemented into a small-sized humanoid robot. The paper reports results of the experiment and discusses about their implications in human-robot interaction research.

\section{Method}

\subsection{Subjects}

The experiment was conducted from October to December, 2008. A total of thirty two persons participated in the experiment.

The number of the elder subjects was fifteen (male: 9, female: 6 , age: $\min 64, \max 79$, mean 69.1). They were inhabitants at a local city in the western area of Japan, and recruited through a survey company. Five thousands yen was paid for each subject.

The number of the younger subjects was seventeen (male: 8 , female: 9 , age: $\min 18$, max 23 , mean 20.8 ). They were university students in the western area of Japan, and recruited with one thousand yen.

\subsection{The Robot Used in the Experiment}

In the experiment, a small-sized humanoid robot shown in Fig. 1 was used. This robot "Robovie-X," which has been developed by Vstone Corporation, stands $34.3 \mathrm{~cm}$ tall and weighs about $1.3 \mathrm{~kg}$. The robot has a total of 17 degrees of freedom (DOFs) at its feet, arms, and head. This large

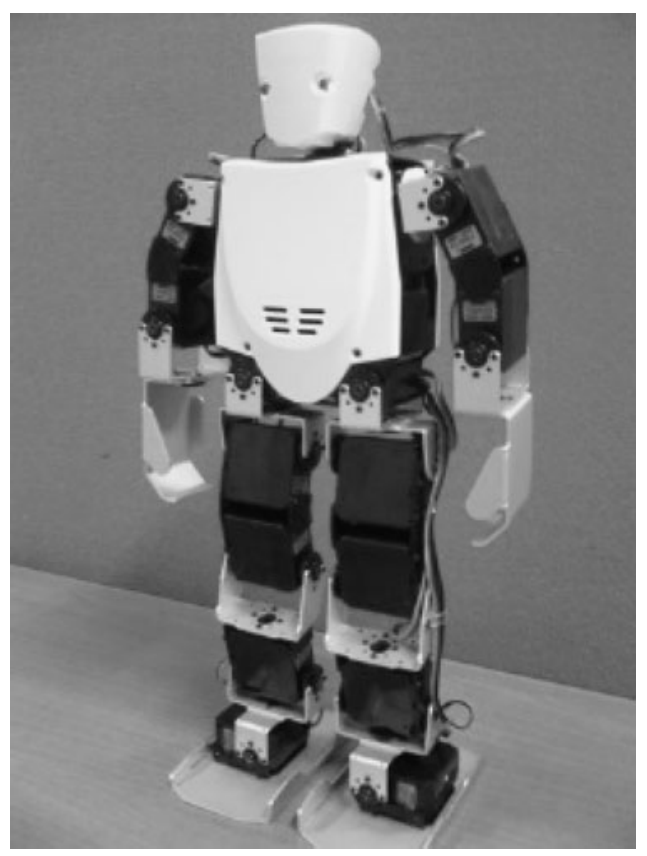

Fig. 1 The robot used in the experiment (Robovie-X) 
Fig. 2 Anger motions by the robot
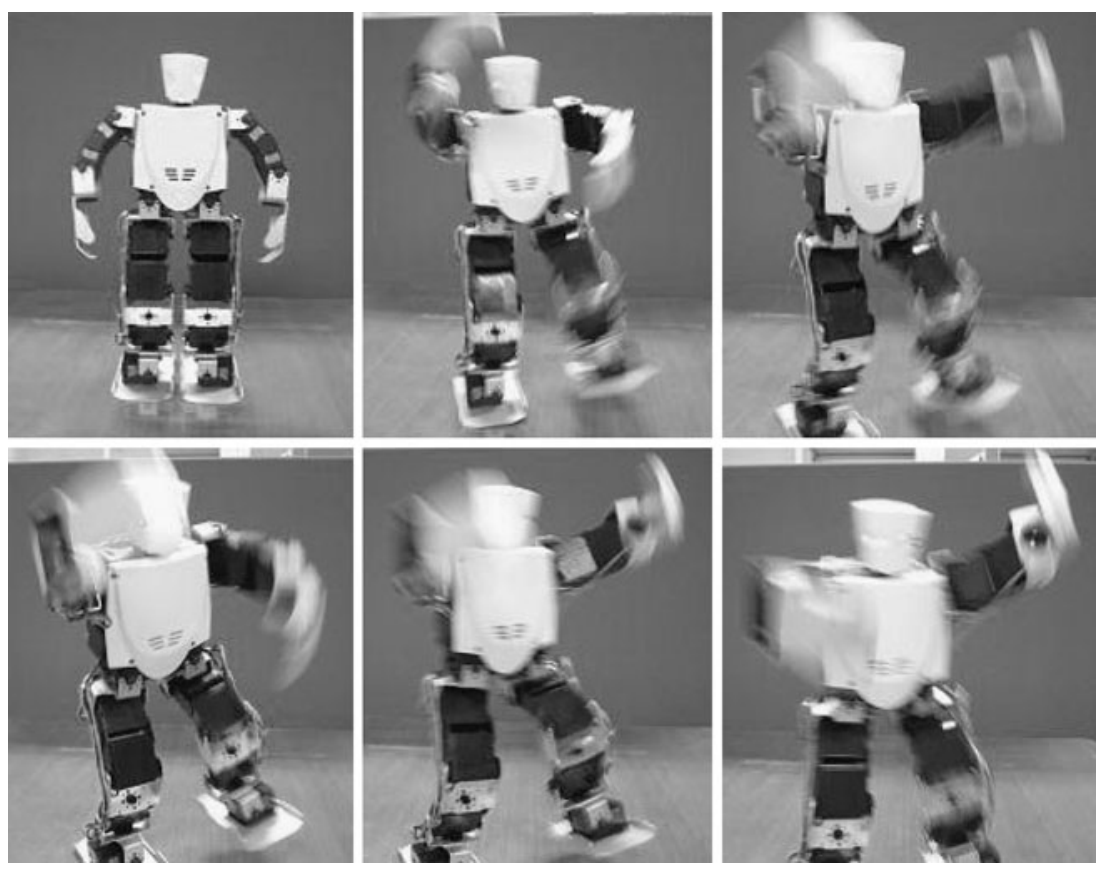

number of DOFs allows it to execute various gestures such as walking, bowing, and a handstand.

Although this robot has a function of utterance based on audio data recorded in advance such as Windows WAV files, this function was not used in the experiment since body motions were the research focus.

\subsection{Affective Body Motions of the Robot}

The research focused on three basic emotions, anger, sadness, and pleasure. These emotions were commonly dealt with in the existing studies on emotion expression by robots [5, 8, 9, 11, 16]. In Wong et al. [17], six emotions of anger, disgust, fear, happiness, surprise, sadness, and neutral were used, and age effects were confirmed for anger, disgust, fear, and sadness. Considering proximity between some emotions [14], disgust and fear were not chosen in the experiment. Moreover, pleasure was added to be compared with the two negative emotions.

Based on some existing studies on affective body motions $[9,11]$ and a literature on modern dances [1], motions corresponding to these emotions were defined as follows:

- Anger: After the robot wave its arms fast and little by little, it marches forward while violently shaking the arms up and down by turns and stepping (like stamping its foot). The speed of the whole motion is high.

- Sadness: The robot bends down a little, and then hides its face with one hand while slowly waving the head on both sides (like wiping its eyes). The speed of the whole motion is low.
- Pleasure: With its open arms, the robot repeats the motion of shaking both the arms up and down (like giving cheers). The speed of the whole motion is high.

The above motions were programmed into the Robovie-X by using the accessory software "RobovieMaker2." Figures 2, 3, 4 show some parts of these affective body motions.

These three body motions by the robot received the agreement among a few university students that they expressed the intended emotions. Since the aim of the experiment was to explore the existence of differences between the elderly and younger people, it was assumed that the validation from one group was sufficient.

\subsection{Procedures}

Each session in the experiment was conducted based on the following procedures.

1. Each subject was explained about the experiment and signed a consent form in a room. In this stage, the experimenters only indicated that the task in the experiment was interaction with a robot or computer.

2. The subject was led to an experiment room, in which the robot was put on a desk, as shown in Fig. 5. After sitting in front of the robot, the subject was more concretely explained about the experiment by the experimenter.

3. First, the subject answered a questionnaire consisting of demographics such as gender, age, and whether he/she had seen real humanoid robots before the experiment. Then, three types of affective body motion were ex- 
Fig. 3 Sadness motions by the robot
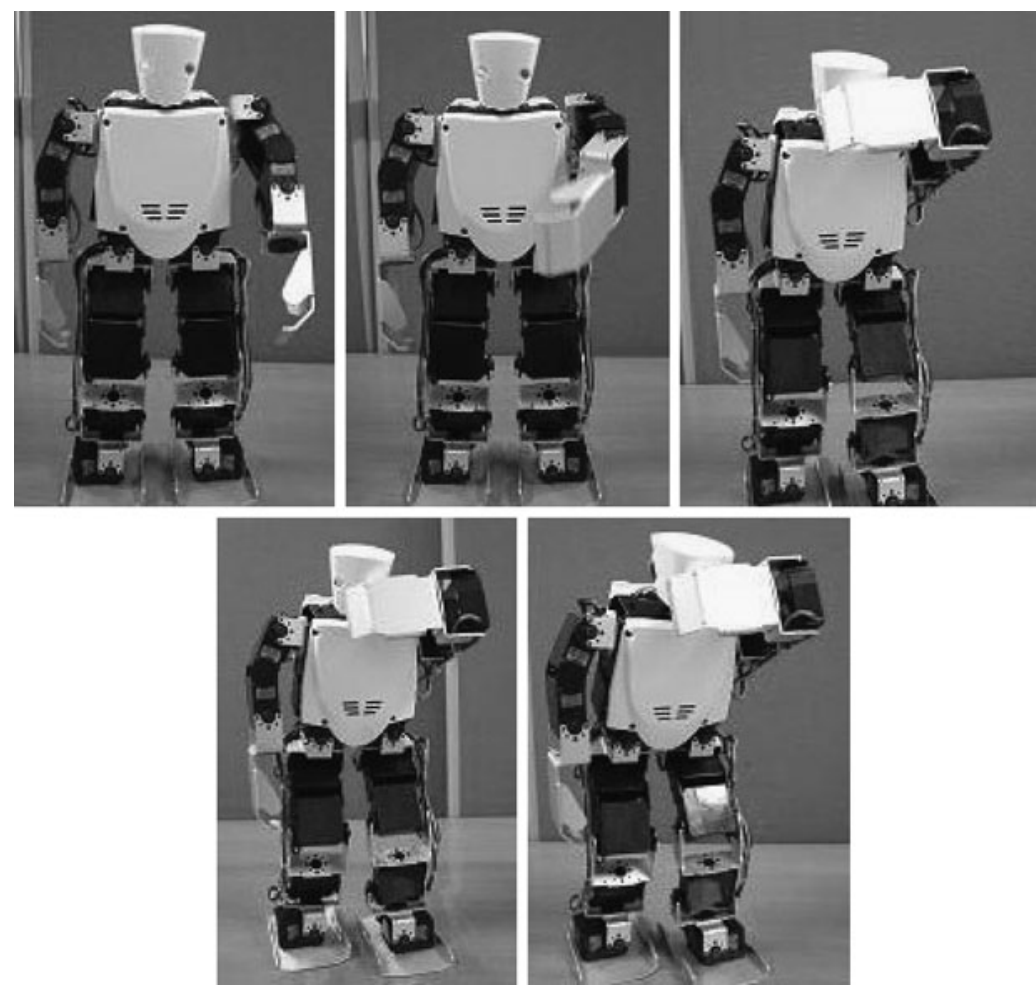

Fig. 4 Pleasure motions by the robot
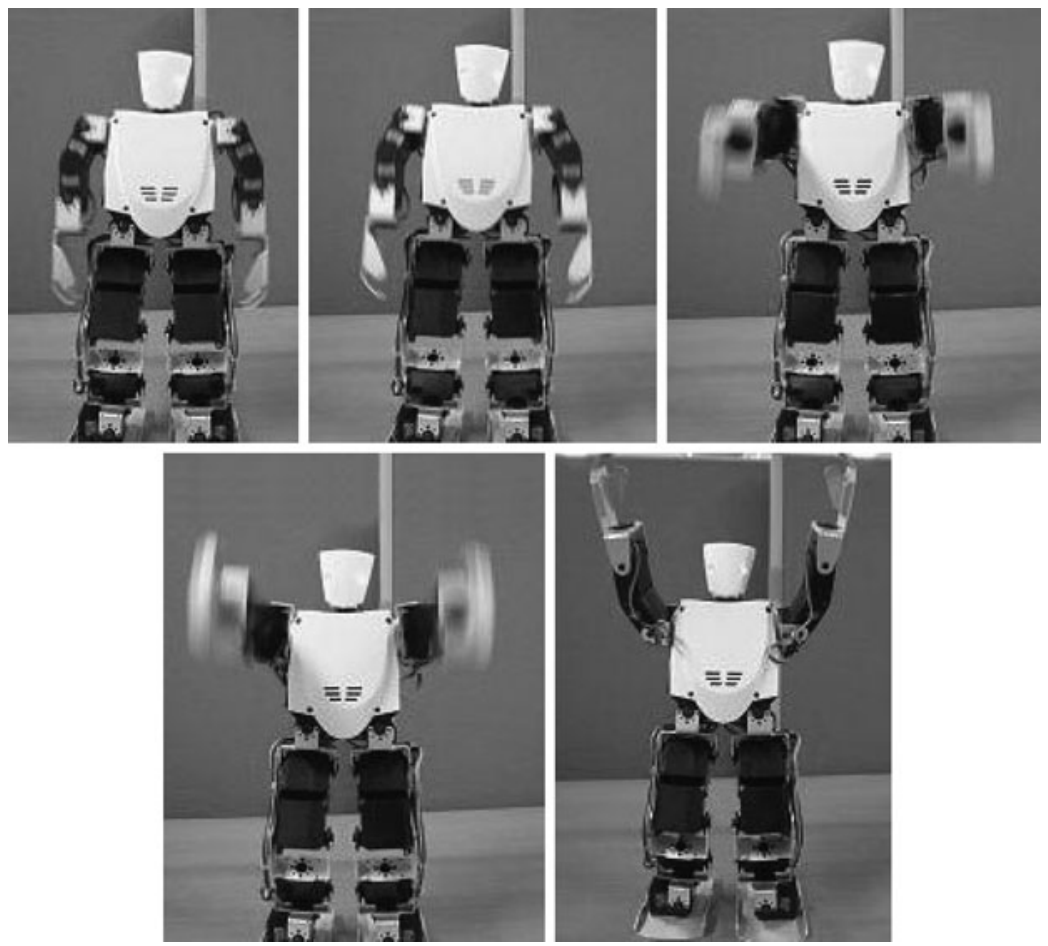

pressed by the Robovie-X in randomized order. Just after each expression, the subject answered a questionnaire to evaluate each body motion.

4. Finally, the experimenter conducted debriefing about the actual aim of the experiment and interview on impressions of robots.

\subsection{Measures}

The measurement in the experiment was based on a selfreported method. The questionnaire for measuring the subjects' evaluation of affective body motions was designed based on the existing study $[9,11]$. For each affective body 
Fig. 5 Overview of the room where the experiment was executed

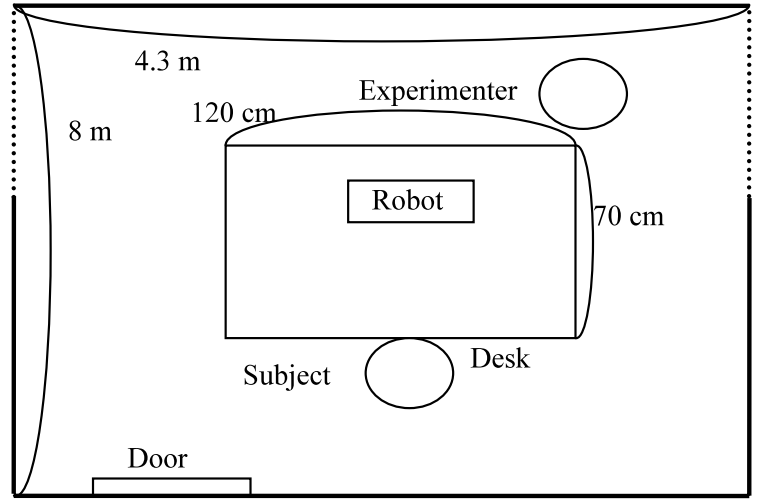

(A view from above)

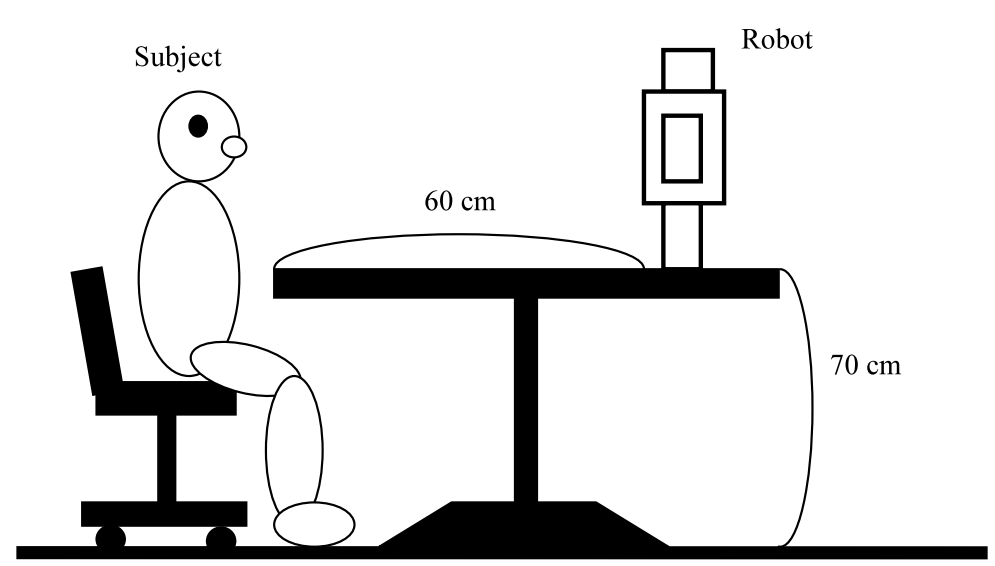

motion expressed by the robot, the questionnaire consisted of three parts; a group of items to measure which emotion and to which degree the subjects felt the expressed motion corresponded to, those to measure which part of the robot motion they paid their attentions to, and those to measure their impression of the magnitude and speed of the motion.

The first part of the questionnaire consisted of seven items corresponding to some basic emotions shown in Table 1 . The subjects were asked to respond to each item to present degrees to which they felt the expressed motion looked like the specified emotion. Each item had a threegraded answer as shown in Table 1. Considering proximity between some emotions [14], the research adopted the way that subjects evaluated plural emotions for one affective body motion.

The second part of the questionnaire consisted of nine items corresponding to the following body and motion parts: head, arms, hands, upper body, legs, feet, speed of motion, magnitude of motion, and others. The subjects were asked to respond to each item to present degrees to which they paid their attentions to the corresponding body or motion part. Each item had a three-graded answer: 1. I paid much attention, 2. I paid a little attention, 3. I paid no attention.

The third part of the questionnaire consisted of two items. One asked the subjects to answer degrees to which they felt the speed of the expressed motion was fast or slow. This item had a five-graded answer (1. I felt it very fast, 2 . I felt it fast, 3. undecided, 4. I felt it slow, 5. I felt it very slow). Another item asked the subjects to answer degrees to which they felt the magnitude of the expressed motion was large or small. This item also had a five-graded answer (1. I felt it very large, 2 . I felt it large, 3. undecided, 4. I felt it small, 5. I felt it very small).

\section{Results}

\subsection{Identification of Affective Body Motions}

First, it was investigated whether there were differences on emotion identification for the body motions by the robot between the elder and student subjects. To simplify the analysis, answers " 1 . I strongly think so" and " 2 . I think so" were coded as 1: "identification as the specified emotion," and others were coded as 0: "no identification as the specified emotion." Table 2 shows the numbers of subjects who identified the presented affective motion as the specified emotions and results of Fisher's exact tests for cross tables based on distinction between students and the elderly. 
Table 1 Item sentences to identify emotions for body motions expressed by the robot

Bold: the emotions that the

\begin{tabular}{lll}
\hline Emotion & Item sentence & Grade of answer \\
\hline Fear & Does the robot look like fearful? & 1. I strongly think so. \\
Pleasure & Does the robot look like pleasant? & 2. I think so. \\
Sadness & Does the robot look like sad? & 3. I do not think so. \\
Hate & Does the robot look like hateful? & \\
Surprise & Does the robot look like surprised? & \\
Anger & Does the robot look like angry? & \\
Others & Does the robot look as if it expresses other emotions? \\
\hline
\end{tabular}

Table 2 Numbers of subjects who identified robot affective body motion as the specified emotions and results of Fisher's exact tests

\begin{tabular}{|c|c|c|c|}
\hline \multicolumn{4}{|c|}{ For anger motion } \\
\hline & Students $(N=17)$ & Elderly $(N=15)$ & Fisher's tests' $p$ \\
\hline Fear & $5(29 \%)$ & $5(33 \%)$ & 1.000 \\
\hline Pleasure & $7(41 \%)$ & $11(73 \%)$ & 0.087 \\
\hline Sadness & $2(12 \%)$ & $3(20 \%)$ & 0.645 \\
\hline Hate & $15(88 \%)$ & $3(20 \%)$ & 0.000 \\
\hline Surprise & $5(29 \%)$ & $9(60 \%)$ & 0.153 \\
\hline Anger & $17(100 \%)$ & $4(27 \%)$ & 0.000 \\
\hline Others & $1(6 \%)$ & $3(20 \%)$ & 0.319 \\
\hline \multicolumn{4}{|c|}{ For sadness motion } \\
\hline & Students $(N=17)$ & Elderly $(N=15)$ & Fishers' tests' $p$ \\
\hline Fear & $8(47 \%)$ & $2(13 \%)$ & 0.060 \\
\hline Pleasure & $0(0 \%)$ & $2(13 \%)$ & 0.212 \\
\hline Sadness & $17(100 \%)$ & $11(73 \%)$ & 0.038 \\
\hline Hate & $11(65 \%)$ & $3(20 \%)$ & 0.016 \\
\hline Surprise & $0(0 \%)$ & $0(0 \%)$ & 1.000 \\
\hline Anger & $0(0 \%)$ & $0(0 \%)$ & 1.000 \\
\hline Others & $4(24 \%)$ & $2(13 \%)$ & 0.659 \\
\hline \multicolumn{4}{|c|}{ For pleasure motion } \\
\hline & Students $(N=17)$ & Elderly $(N=15)$ & Fishers' tests' $p$ \\
\hline Fear & $0(0 \%)$ & $1(7 \%)$ & 0.469 \\
\hline Pleasure & $16(94 \%)$ & $12(80 \%)$ & 0.319 \\
\hline Sadness & $1(6 \%)$ & $2(13 \%)$ & 0.589 \\
\hline Hate & $1(6 \%)$ & $1(7 \%)$ & 1.000 \\
\hline Surprise & $9(53 \%)$ & $3(20 \%)$ & 0.076 \\
\hline Anger & $2(12 \%)$ & $1(7 \%)$ & 1.000 \\
\hline Others & $4(24 \%)$ & $2(13 \%)$ & 0.659 \\
\hline
\end{tabular}

For the body motion of anger, the rates of those who identified it as anger and hate in the student subject group (88\% and $100 \%$ respectively) were larger than those in the elderly subject group (20\% and $27 \%$ respectively) at statistically significant levels. For the body motion of sadness, the rates of those who identified it as sadness and hate in the student subject group (100\% and 65\% respectively) were larger than those in the elderly subject group (73\% and 20\% respectively) at statistically significant levels. These results showed that more subjects in the younger group accurately identified the anger and sadness motions of the robot as the intended ones or proxy ones, than in the elder group.

For the body motion of pleasure, there was no statistically significant difference on identification of the specified emotions between these groups, and many subjects in both younger and elder groups accurately identified the pleasure motion of the robot as pleasure (younger: $94 \%$, elderly: $80 \%)$.

\subsection{Attention to Body and Motion Parts}

Second, it was investigated whether there were differences between the elder and student subjects on body and motion parts they paid their attentions to. To simplify the analysis, answers " 1 . I paid much attention" and " 2 . I paid attention a little" were coded as 1: "attention to the specified part," and others were coded as 0: "no attention to the specified part." Table 3 shows the numbers of subjects who paid their attentions to the specified part of body and motion for each affective motion and results of Fisher's exact tests for cross tables based on distinction between students and the elderly.

For the body motion of anger, the rate of those who paid their attentions to the upper body of the robot in the student subject group (53\%) was smaller than that in the elderly subject group (93\%) at a statistically significant level. This result showed that more subjects in the elder group paid their attention to the upper body of the robot expressing the anger motion, than in the younger group.

For the body motion of sadness, the rates of those who paid their attentions to the legs, feet, magnitude of motion, and other parts of the robot in the student subject group $(35 \%, 18 \%, 53 \%$ and $0 \%$ respectively) were smaller than those in the elderly subject group $(80 \%, 67 \%, 93 \%$ and $33 \%$ respectively) at statistically significant levels. These results showed that more subjects in the elder group paid their attention to the legs, feet, magnitude of motion, and other parts of the robot expressing the sadness motion, than in the younger group. 
Table 3 Numbers of subjects who paid attentions to the specified part of body and motion for each affective body motion of the robot and results of Fisher's exact tests

\begin{tabular}{lccc}
\hline For anger motion & & & \\
\hline & $\begin{array}{c}\text { Students } \\
(N=17)\end{array}$ & $\begin{array}{c}\text { Elderly } \\
(N=15)\end{array}$ & $\begin{array}{c}\text { Fishers' } \\
\text { tests' } p\end{array}$ \\
\hline Head & $5(29 \%)$ & $9(60 \%)$ & 0.153 \\
Arms & $15(88 \%)$ & $15(100 \%)$ & 0.486 \\
Hands & $17(100 \%)$ & $15(100 \%)$ & 1.000 \\
Upper body & $9(53 \%)$ & $14(93 \%)$ & 0.018 \\
Legs & $17(100 \%)$ & $15(100 \%)$ & 1.000 \\
Feet & $17(100 \%)$ & $15(100 \%)$ & 1.000 \\
Speed of motion & $17(100 \%)$ & $15(100 \%)$ & 1.000 \\
Magnitude of motion & $17(100 \%)$ & $15(100 \%)$ & 1.000 \\
Others & $3(18 \%)$ & $5(33 \%)$ & 0.423 \\
\hline
\end{tabular}

For sadness motion

\begin{tabular}{lcll}
\hline & $\begin{array}{c}\text { Students } \\
(N=17)\end{array}$ & $\begin{array}{l}\text { Elderly } \\
(N=15)\end{array}$ & $\begin{array}{l}\text { Fishers' } \\
\text { tests' } p\end{array}$ \\
\hline Head & $16(94 \%)$ & $14(93 \%)$ & 1.000 \\
Arms & $17(100 \%)$ & $15(100 \%)$ & 1.000 \\
Hands & $17(100 \%)$ & $14(93 \%)$ & 0.469 \\
Upper body & $14(82 \%)$ & $14(93 \%)$ & 0.603 \\
Legs & $6(35 \%)$ & $12(80 \%)$ & 0.016 \\
Feet & $3(18 \%)$ & $10(67 \%)$ & 0.010 \\
Speed of motion & $10(59 \%)$ & $13(87 \%)$ & 0.122 \\
Magnitude of motion & $9(53 \%)$ & $14(93 \%)$ & 0.018 \\
Others & $0(0 \%)$ & $5(33 \%)$ & 0.015 \\
\hline
\end{tabular}

For pleasure motion

\begin{tabular}{lcll}
\hline & $\begin{array}{l}\text { Students } \\
(N=17)\end{array}$ & $\begin{array}{l}\text { Elderly } \\
(N=15)\end{array}$ & $\begin{array}{l}\text { Fishers' } \\
\text { tests' } p\end{array}$ \\
\hline Head & $7(41 \%)$ & $11(73 \%)$ & 0.087 \\
Arms & $17(100 \%)$ & $15(100 \%)$ & 1.000 \\
Hands & $14(82 \%)$ & $15(100 \%)$ & 0.229 \\
Upper body & $15(88 \%)$ & $15(100 \%)$ & 0.486 \\
Legs & $16(94 \%)$ & $12(80 \%)$ & 0.319 \\
Feet & $11(65 \%)$ & $10(67 \%)$ & 1.000 \\
Speed of motion & $15(88 \%)$ & $14(93 \%)$ & 1.000 \\
Magnitude of motion & $17(100 \%)$ & $14(93 \%)$ & 0.469 \\
Others & $1(6 \%)$ & $2(13 \%)$ & 0.589 \\
\hline
\end{tabular}

For the body motion of pleasure, there was no statistically significant difference on attentions to the specified parts of body and motion between these groups.

\subsection{Impression of Motion Speed and Magnitude}

Third, it was investigated whether there were differences on impression of the magnitude and speed of the motion be-
Table 4 Means and standard deviations of motion speed and magnitude impression scores, and results of ANOVAs

\begin{tabular}{|c|c|c|c|c|}
\hline \multicolumn{5}{|c|}{ Impression of motion speed } \\
\hline & \multicolumn{2}{|c|}{ Students } & \multicolumn{2}{|c|}{ Elderly } \\
\hline & Mean & $\overline{S D}$ & Mean & $S D$ \\
\hline Anger & 1.5 & 0.7 & 1.1 & 1.0 \\
\hline Sadness & -0.9 & 0.8 & -0.1 & 0.9 \\
\hline Pleasure & 0.7 & 0.9 & 0.6 & 1.0 \\
\hline Age: & \multicolumn{4}{|c|}{$F(1,30)=0.192$} \\
\hline Motion: & \multicolumn{4}{|c|}{$F(2,60)=33.801^{\mathrm{c}}$} \\
\hline Interaction: & \multicolumn{4}{|c|}{$F(2,60)=3.719^{\mathrm{a}}$} \\
\hline
\end{tabular}

Impression of motion magnitude

\begin{tabular}{|c|c|c|c|c|}
\hline & \multicolumn{2}{|c|}{ Students } & \multicolumn{2}{|c|}{ Elderly } \\
\hline & Mean & $S D$ & Mean & $S D$ \\
\hline Anger & 1.5 & 0.6 & 0.4 & 1.2 \\
\hline Sadness & -0.2 & 1.0 & -0.1 & 1.2 \\
\hline Pleasure & 1.3 & 0.6 & 0.7 & 1.3 \\
\hline Age: & \multicolumn{4}{|c|}{$F(1,30)=24.099^{\mathrm{a}}$} \\
\hline Motion: & \multicolumn{4}{|c|}{$F(2,60)=16.378^{c}$} \\
\hline Interaction: & \multicolumn{4}{|c|}{$F(2,60)=3.825^{\mathrm{a}}$} \\
\hline
\end{tabular}

${ }^{\mathrm{a}} p<0.05$

${ }^{\mathrm{b}} p<0.01$

${ }^{\mathrm{c}} p<0.001$

tween the elder and student subjects. For this aim, the scores of the speed impression item were coded within the range from -2 to 2 ( -2 : "I felt it very slow," -1 : "I felt it slow," 0: "undecided," 1: "I felt it fast," 2: "I felt it very fast"). Moreover, the scores of the magnitude impression item were also coded within the range from -2 to 2 (-2: "I felt it very small," -1: "I felt it small," 0: "undecided," 1: "I felt it large," 2: "I felt it very large"). Then, two-way mixed ANOVAs were performed for these scores to confirm the main effects of age and motion, and their interaction effects. Table 4 shows the means and standard deviations of the scores and results of the ANOVAs. Moreover, Fig. 6 visualizes the means of these impression scores based on motion types and student/elderly conditions.

The results found a statistically significant main effect of age in the magnitude impression scores. Moreover, they found statistically significant main effects of affective body motions in both speed and magnitude impression scores. Furthermore, there were statistically significant interaction effects of age and motion in both the speed and magnitude impression scores. Post-hoc tests with Bonferroni's method found that: for the sadness motion the speed impression scores in the student subjects were lower than those in the elderly subjects, in the student subjects the speed impression 
Fig. 6 Means of speed and magnitude impression scores
Impression of Motion Speed

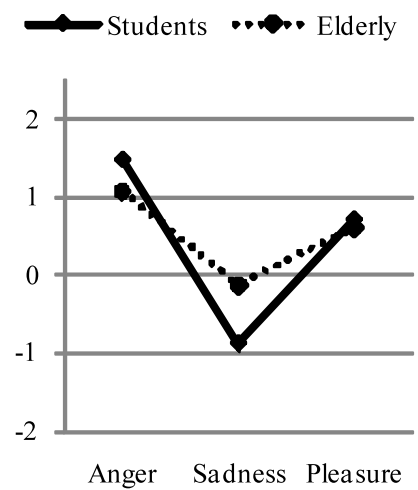

Impression of Motion Magnitude

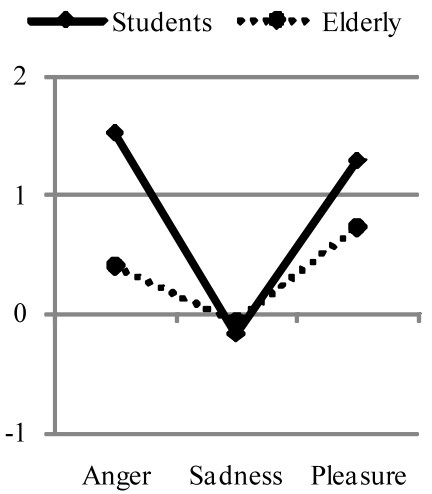

Table 5 Correlation coefficients between items of emotion identification, attention to body parts, and impression of motions
${ }^{\mathrm{a}} p<0.1$

${ }^{\mathrm{b}} p<0.05$

\begin{tabular}{lllc}
\hline For anger motion & & & \\
\hline & Kind of correlation & Judgment as hate & Judgment as anger \\
\hline Attention to upper body & $\psi$ & -0.131 & $-0.306^{\mathrm{a}}$ \\
Magnitude impression & Point biserial & $0.405^{\mathrm{b}}$ & $0.302^{\mathrm{a}}$ \\
\hline For sadness motion & & & \\
\hline & Kind of correlation & Judgment as sadness & Judgment as hate \\
\hline Attention to legs & $\psi$ & -0.143 & 0.016 \\
Attention to feet & $\psi$ & -0.265 & -0.088 \\
Attention to motion magnitude & $\psi$ & -0.236 & 0.131 \\
Attention to other parts & $\psi$ & $-0.358^{\mathrm{b}}$ & -0.206 \\
Speed impression & Point biserial & -0.328 & $-0.319^{\mathrm{a}}$ \\
\hline
\end{tabular}

scores for the sadness motion was lower than those for the other motions, and in the elderly subjects the scores for the sadness motion was lower than those for the angry motion. Moreover, the analyses found that: for the anger motion the magnitude impression scores in the student subjects were higher than those in the elderly subjects, and in the student subjects the magnitude impression scores for the sadness motion was lower than those for the other motions. There was no statistically significant difference in the other comparisons.

\subsection{Correlations between Emotion Identification, Attention to Body Parts, and Impression of Motions}

Finally, correlation analyses were performed to investigate relationships between the subject's emotion identification for, their attentions to body and motion parts for, and their impressions of the affective body motions by the robot. These correlation coefficients were calculated between the items showing differences between the student and elder subjects. Table 5 shows the correlation coefficients. The values between the items of emotion identification and attention to body and motion parts mean $\psi$-coefficients, and those between the items of emotion identification and motion impression mean point biserial correlation coefficients, respectively.

For the anger motion, the identification as hate and anger were positively correlated with the magnitude impression at moderate levels. The identification as anger was also negatively correlated with the attention to upper body at a moderate level. For the sadness motion, the identification as sadness and hate were negatively correlated with the speed impression at moderate levels. The identification as sadness was also negatively correlated with the attention to other parts at a moderate level.

Moreover, it was investigated whether the subjects' experiences of humanoid robots related with the above emotion identification and attention to body and motion parts. About a half of the subjects reported that they had seen real humanoid robots (students: 8/17, elderly: 7/15). However, this type of experiences had no statistically significant correlation with emotion identification and attention to body and motion parts (correlation coefficients between -0.280 and -0.012 , n.s.), except of the one with identification of the sadness motion as hate $(\psi=-0.323, p<0.1)$. 


\section{Discussion}

\subsection{Findings}

On the research question 1: "Are there differences on emotion identification of body motions expressed by robots between younger and elder people?" it was shown that almost all the student subjects identified the three types of affective body motion as the emotions intended by the motions, and many of them identified the anger and sadness motions of the robot as hate, which is an emotion proximate to anger and sadness [14], in comparison that many of the elder subjects did not identify these motions as either the intended ones or the proximate one. On the other hand, there was no difference on identification of the pleasure motion between these subject groups. These results are consistent with the results of Wong et al. [17], in case of human facial emotion expressions.

Moreover, in comparison with the student subjects, more of the elder subjects paid their attentions to the upper body in the anger motion of the robot, the legs and feet in the sadness motion. These parts of the robot body are the ones not important on the affective expression implemented in the experiment. These results are also consistent with the results of Wong et al. [17], in case of human facial emotion expressions, in the sense that the elder subjects paid less attentions to parts required for identifying the intended emotions than did the younger subjects. Furthermore, in comparison with the elder subjects, the student subjects more strongly felt that the magnitude of the anger motion by the robot was large, and the speed of the sadness motion was slow.

On the research question 2: "Are there correlations between the accuracy of the emotion identification and cognitive bias to robots' specific body motion parts?" the correlation coefficient between the items of identification as anger and attention to upper body showed that identification for the anger motion of the robot was more correct as the upper body was less paid attention to. The correlation coefficients also showed that identification for the anger motion of the robot was more correct as the magnitude impression was stronger, and identification of the sadness motion was more correct as the speed impression was weaker. These results are also consistent with the results of Wong et al. [17], in case of human facial emotion expressions, suggesting that the tendency of cognitive bias on parts not important for identifying certain emotions adversely affected the older subjects' ability to identify these emotions.

\subsection{Implications}

The experiment of the paper can be interpreted as a case study on a gap between robotics designers and users in
Japan. The experimenters, who were not the elderly, implemented the affective body motions of the robot while interpreting results of the existing studies and receiving the agreement among a few university students that the intended emotions were expressed. However, the experimenters' and younger evaluators' subjective factors on affective expression, dependent on their culture and cognitive characteristics different from the elderly, may be incorporated into the implementation process, and it may lead to the differences on evaluation of the motions between the student and elder subjects.

The above interpretation has some important implications. Robotics designers using body motions as a mode of affective human-robot interaction should be sensitive for human factors for emotion identification and their dependence on users' demographics such as age. In other words, it should sufficiently be investigated which physical characteristics can influence emotion identification of body motions for a specific group of users. In particular, when users in human-robot interaction are the elderly, robotics designers should consider combination of affective body motions with other modes such as speech and language.

Moreover, the experimental results in the paper suggested age differences on emotion identification even in one culture. There may be cultural differences on emotional body expressions [3,6], and several interaction effects between ages, gender, and so on. If robotics designers consider multicultural applications of affective body motions, they should consider adaptation mechanisms for different body motion rules of emotion expression.

\subsection{Limitations}

Since we only tested with a particular type of robots with a particular interaction with subjects from specific groups, the generality of our findings is limited. We believe that they are applicable to interactions with a robot of similar size and appearance, and interactions of similar complexity. Nevertheless, our current research has some problems.

First, the affective body motions used in the experiment were implemented along qualitative guidelines that were extracted from the existing studies, and were sufficiently not validated. It was shown that the younger subjects accurately identified these motions as the intended ones, and it limits their validity to some extent. In this sense, the research in the paper is within case studies.

Second, the human-robot interaction in the experiment was one way from the small-sized humanoid robot to the human subjects without any context and concrete task. Goetz, Kiesler, and Powers [4] and Kidd and Breazeal [7] found effects of robot appearance under concrete physical tasks of subjects. Moreover, Mutlu et al. [10] found interaction effects between gender and task structures of cooperation or 
competition with robots. Furthermore, Scopelliti, Giuliani, and Fornara [15] found age difference of familiarity with robots in the context of domestic use. The experiment in the paper did not take into account interaction effects between user demographics, contexts where robots were used, other physical characteristics of robots such as size and appearance, as dealt with in these studies. In particular, domestic use for elderly care should be considered to validate the usefulness of body expression in interaction of robots with the elder people since their interaction effect may improve the accuracy of emotion identification of the elderly.

Third, the total number of subjects in the experiment was not sufficient. Moreover, our control of the experimental conditions was also insufficient, for example, the usage of different experiment rooms (with and without windows) and consideration of elder subjects' cognitive (auditory and visual) characteristics. In particular, users' cognitive characteristics should be cared on screening subjects for more strict experimental designs.

Fourth, the experiment did sufficiently not take into account other demographic factors such as gender, experience of robots, educational background, and psychological construct. Some existing studies suggested influences of attitude and anxiety toward robots into behaviors toward them $[12,13]$. Although the experiment found no relationship of robot experience with emotion identification and impression, it was found that experiences of robots affects these psychological constructs. Thus, experiences of robots may indirectly affect emotion identification and impression. Moreover, gender and educational backgrounds may influence experiences of robots. These variables should be considered.

The aforementioned problems must be tackled in future experiments by extending the experimental design, for example, by sampling from more groups and using several types of robots, tasks, and demographic variables.

\section{Conclusions}

Based on a psychological research about age dependence on emotion identification in human facial expressions, the study in the paper conducted a psychological experiment in Japan to investigate age dependence in identification of affective body expressions of robots, by using a small-sized humanoid robot. In the experiment, three types of affective motion expression (anger, sadness, and pleasure) were implemented on the robot. The results of the experiment, which consisted of seventeen university student subjects and fifteen elder subjects, showed differences between younger and elder subjects on emotion identification, body parts paid attention to, and impressions of motion speed and magnitude for these affective body motions of the robot. Moreover, the results suggested correlations between the accuracy of emotion identification and cognitive bias to the robot's specific body motion parts. Based on these results, the paper discussed about some implications in human-robot interaction research, in particular, designers' sensitivity for human factors for emotion identification and their dependence on users' demographics such as age.

Further experiments are going to be conducted with extension of experimental design, including more validated motions of robots, contextualized interaction, wider sampling, and more demographic variables.

Acknowledgement The authors deeply thank Dr. Atsunobu Suzuki of Nagoya University for his providing us with research information on age differences on emotion identification of human facial expression. The work was supported in part by the High-Tech Research Center Project for Private Universities with a matching fund subsidy from Ministry of Education, Culture, Sports, Science and Technology (MEXT), 2002-2006.

Open Access This article is distributed under the terms of the Creative Commons Attribution Noncommercial License which permits any noncommercial use, distribution, and reproduction in any medium, provided the original author(s) and source are credited.

\section{References}

1. Anderson J (1986) Ballet and modern dance: a concise history. Princeton Book Company, Princeton

2. Breazeal C (2003) Emotion and sociable humanoid robots. Int J Hum-Comput Stud 59:119-155

3. Cornelius RR (1996) The science of emotion: research and tradition in the psychology of emotions. Prentice-Hall, New York

4. Goetz J, Kiesler S, Powers A (2003) Matching robot appearance and behaviors to tasks to improve human-robot cooperation. In: Proc 12th IEEE int workshop robot hum Interact Commun, pp $55-60$

5. Ito K, Miwa H, Matsumoto M, Zecca M, Takanobu H, Roccella S, Carrozza MC, Dario P, Takanishi A (2004) Various emotional expressions with emotion expression humanoid robot WE-4RII. In: Proc 1st IEEE technical exhibition based conf robotics and automation, pp 35-36

6. Kemper TD (ed) (1990) Research agendas in the sociology of emotions. SUNY Press, Albany

7. Kidd C, Breazeal C (2004) Effect of a robot on user perceptions. In: Proc IEEE/RSJ int conf intell robots syst, pp 3559-3564

8. Kim EH, Kwak SS, Han J, Kwak YK (2009) Evaluation of the expressions of robotic emotions of the emotional robot, "Mung". In: Proc the 3rd int conf ubiquitous information management and communication, pp 362-365

9. Marui N, Matsumaru T (2005) Emotional motion of humanfriendly robot: emotional expression with bodily movement as the motion media. In: 23th annual conference of robotics society of japan (in Japanese)

10. Mutlu B, Osman S, Forlizzi J, Hodgins J, Kiesler S (2006) Task structure and user attributes as elements of human-robot interaction design. In: Proc 15th IEEE int symp robot hum interact commun, pp 74-79

11. Nakata T, Mori T, Sato T (2001) Quantitative analysis of impression of robot bodily expression based on Laban movement theory. J Robot Soc Jpn 19:252-259 (in Japanese) 
12. Nomura T, Suzuki T, Kanda T, Kato K (2006) Measurement of negative attitudes toward robots. Interact Stud 7:437-454

13. Nomura T, Kanda T, Suzuki T, Kato K (2008) Prediction of human behavior in human-robot interaction using psychological scales for anxiety and negative attitudes toward robots. IEEE Trans Robot 24:442-451

14. Plutchik R (1984) Emotion: A general psychoevolutionary theory. In: Scherer KR, Ekman P (eds) Approaches to emotion. Erlbaum, Hillsdale, pp 197-219

15. Scopelliti M, Giuliani MV, Fornara F (2005) Robots in a domestic setting: a psychological approach. Univers Access Inf Soc 4:146155

16. Yuk N-S, Kwon D-S (2008) Realization of expressive body motion using leg-wheel hybrid mobile robot: KaMERo. In: Proc int conf control, automation and systems, pp. 2350-2355

17. Wong B, Cronin-Golomb A, Neargarder S (2005) Patterns of visual scanning as predictors of emotion identification in normal aging. Neuropsychology 19:739-749
Tatsuya Nomura received his B.S. degree and M.S. degree in mathematics from Osaka University, Osaka, Japan, in 1987 and 1989, respectively. He was with the Corporate Research and Development Group at Sharp Corporation from 1989 to 2000, and received his D.E. degree from Kyoto University, Kyoto, Japan, in 1998. He was with Hannan University, Osaka, Japan, from 2000 to 2004. He is currently an associate professor in the Department of Media Informatics, Ryukoku University, Shiga, Japan, and a visiting researcher at the Intelligent Robotics and Communication Laboratories at ATR (Advanced Telecommunications Research Institute), Kyoto, Japan. His research interests include user studies in human-robot interaction and affective computing.

Akira Nakao received his B.E. degree in media informatics from Ryukoku University, Shiga, Japan, in 2009. He is currently with the Graduate School of Science and Technology, Ryukoku University, Shiga, Japan. His research interests include affective computing and image processing. 